\title{
Concevoir Une Société Yacouba Sans La Pratique De L’excision, Une Équation Impossible?
}

\author{
Kouadıo Kouassi Kan Adolphe
}

Attaché de rherche au CRD/

Université Alassane Ouattara de Bouaké, Côte d'Ivoire

Doudou Dimi Thédore

Chargé de recherche au CRD/

Université Alassane Ouattara de Bouaké, Côte d'Ivoire

Tuo Donoukporo

Attaché de rherche au CRD/

Université Alassane Ouattara de Bouaké, Côte d’Ivoire

doi: 10.19044/esj.2017.v13n8p226 URL:http://dx.doi.org/10.19044/esj.2017.v13n8p226

\begin{abstract}
The practice of violence against women is becoming increasingly acute in Côte d'Ivoire. The forms and frequencies vary from one cultural area to another. Thus, in the west, in the Tonkpi, female genital mutilation (FGM), occupy the heights of forms of gender-based violence (GVA), (CRD, 2016). In order to identify the strategies of circumcisers in this period of prohibition of FGM and the representations that underpin this practice, in spite of national and international policies, research was carried out in three sub-prefectures of the department of Man and Biankouma from February to March 2016. This is a qualitative study. The results confirmed that the attachment to custom and the adoption of new strategies are the factors explaining the persistence of the practice of excision in Côte d'Ivoire.
\end{abstract}

Keywords: Violence against women; Female genital mutilation; Excision; Socio-cultural practices; Region of Tonkpi

\section{Résumé}

La pratique des violences contre les femmes se pose avec acuité en Côte d'Ivoire. Les formes et les fréquences varient d'une aire culturelle à une autre. Ainsi, à l'ouest, dans le Tonkpi, les mutilations génitales féminines (MGF), occupent les sommets des formes de violence basée sur le genre (VBG), (CRD, 2016). En vue d'identifier les stratégies des exciseuses en cette période d'interdiction des MGF et les représentations qui sous-tendent cette pratique, en dépit des politiques nationales et internationales, une 
recherche s’est effectuée dans trois sous-préfectures du département de Man et de Biankouma de février à mars 2016. Il s’agit d'une étude qualitative. Les résultats ont confirmé que l'attachement à la coutume et l'adoption de nouvelles stratégies sont les facteurs explicatifs de la persistance de la pratique de l'excision en Côte d'Ivoire.

Mots-clés: Violences contre la femme ; mutilations génitales féminines ; excision ; pratiques socioculturelles ; région du Tonkpi

\section{Introduction}

En Côte d'Ivoire la violence basée sur le genre est devenue un véritable phénomène social capable d'entraver tous les efforts de développement durable car, touchant principalement la majorité de la population constituée par les femmes ainsi que les jeunes filles ((Heise et al, 1999 ; Guedes, 2004 ; UNFPA, 2008).

Ces violences se manifestent sous diverses formes et constituent des violations des droits humains. Il s’agit entre autre du viol, de l'agression sexuelle et physique, du mariage forcé, du déni de ressources, d'opportunité ou de service, de la maltraitance psychologique ainsi qu'émotionnelle.

Plusieurs dispositions internationales existent pour lutter contre les violences basées sur le genre(VGB). Citons à titre d'exemple la Convention et le Protocole Additionnel sur l'Elimination de toutes les formes de discrimination à l'égard des femmes, la Déclaration des Nations-Unies sur l'Elimination de la violence contre les femmes, les dispositions de la Plateforme de la conférence de Beijing de 1995 sur les violences et sur la protection des femmes, les dispositions de la Déclaration du Millénaire sur les formes de violences à l'encontre des femmes, la Résolution des NationsUnies sur « L'Intensification des Efforts pour éliminer toutes les Formes de violences à l'Egard des Femmes », la campagne visant à l'éradication de la violence faite aux femme d'ici 2015, la Déclaration Solennelle sur l'Egalité de Genre en Afrique, etc.

A l'instar de certains pays du monde, la Côte d'Ivoire s'est engagée à lutter contre toutes les formes de violences basées sur le genre depuis plusieurs années. Elle a notamment adhéré aux instruments internationaux consacrant l'égalité entre les hommes et les femmes et la non-discrimination des femmes tels que la Convention sur l'Elimination de toutes les formes de Discrimination à l'Egard des Femmes (CEDEF). Elle a également mis en place un cadre légal et institutionnel favorable à la mise en œuvre de ces instruments. A ce titre, le pays dispose d'un « Ministère de la Solidarité de la famille de la Femme et de l'Enfant» et d'une Direction Générale de la Promotion de la Femme et de l'Egalité des Genres en son sein. Egalement, de nombreuses interventions ont étés entreprises sur l'ensemble du territoire 
dans plusieurs domaines (santé, juridique, psychosociale, protection et sécurité) par un large éventail d’acteurs, issus des structures étatiques, de la société civile nationale, des ONG nationales ou internationales, des organismes du Système des Nations Unies. Cependant, les violences faites aux femmes et aux filles continuent d’avoir des conséquences désastreuses sur les individus, les familles et les communautés. Ces violences se manifestent de diverses manières et se poursuivent à travers des pratiques socioculturelles quotidiennes pour s'étendre à des pratiques systématiques et institutionnelles qui limitent la vie et les opportunités des femmes et des filles. Ainsi, au sein des communautés, notamment en milieu rural, les hommes continuent d'avoir le contrôle des ressources et sont les auteurs des actes des violences infligées aux femmes et aux filles.

Au titre des Mutilations Génitales Féminines (MGF), la Côte d'Ivoire est l'un des pays les plus touchés en Afrique de l'Ouest. Les résultats préliminaires de l'enquête à indicateurs multiples (MICS 2012) estiment à environ 38 \% la prévalence de l'excision en Côte d'Ivoire, avec des disparités régionales allant jusqu'à $87 \%$ au Nord et $73 \%$ à l'Ouest du pays. Pratiquées dans la plupart des groupes culturels, les mutilations génitales féminines bénéficient encore d’un large consensus social.

Selon le rapport 2012 de la plateforme de services VBG du District des Montagnes, à l'ouest de la Cote d'ivoire, on a assisté à 987 cas de VBG (335 cas de violences sexuelles, 283 agressions physiques, 18 mariages forcés, 351 autres types de VBG).

Par ailleurs, selon le Bulletin Humanitaire OCHA Côte d’Ivoire N¹ 2014, le nombre de cas rapportés de violences sexuelles en 2013 était de 776 pendant l'année 2011 et de 731 en 2012.

En outre, rapporte le document, on assiste à des vagues successives de cérémonies d'excision.

Une récente résolution de l’Assemblée générale des Nations Unies, relative à l'intensification des efforts mondiaux en vue d'éradiquer les MGF, adoptée le 20 décembre 2012 demande instamment aux pays de condamner toutes les pratiques dangereuses qui affectent les femmes et les filles, en particulier les mutilations génitales féminines, et de prendre toutes les mesures nécessaires, y compris l'application de la législation, la sensibilisation et l'allocation de ressources suffisantes, pour protéger les femmes et les filles contre cette forme de violence.

Si les différentes actions de sensibilisation semblent fléchir l'attitude de certaines populations (engagement verbal des chefs de communauté à lutter contre la pratique des MGF), à l'Ouest de la Côte d'Ivoire, dans le Tonkpi, les luttes buttent contre une « farouche » résistance (Opadou, Traoré, 2015 ; rapport du sous-préfet, de la plate forme des VBG de Man, 2016). 
En effet dans les sous-préfectures de Sandougou Soba, Kpata, et Gouiné, les populations, au nom de la coutume, excisent encore les filles. Si la pratique ne se fait plus au vu et au su du grand public (avec de « grandes et longues cérémonies festives »), les nouvelles stratégies adoptées et la création de nouveaux sites permettent aux exciseuses de continuer leur activité.

La présente étude cherche à identifier les stratégies des exciseuses en cette période d'interdiction des MGF et les représentations sociales qui soutiennent cette pratique.

\section{Méthode}

Il s’agit de présenter le site de l'étude, l'échantillonnage et la méthode d'analyse. La présente recherche est circonscrite dans la région du Tonkpi, précisément dans les sous-préfectures de Kpata, Sandougou-Soba et gouiné. Elles sont peuplées d'autochtones Yacouba et Toura. On rencontre toutefois, dans certains villages de la sous-préfecture de Gouiné des allogènes Burkinabé, Maliens et des allochtones baoulé (CRD, 2016).

Le choix du site a été suscité par le constat de la persistance de la pratique de l'excision lors d'une étude de terrain commanditée par le Centre de Recherche pour le Développement (CRD) de février à mars 2016 dans la région du Tonkpi. L’étude a porté sur les violences basées sur le genre (VBG) en général, mais la pratique de l'excision a marqué notre attention, vue l’importance que la population lui accorde et les stratégies mobilisées pour la pratiquer en cette période d'interdiction des MGF en Côte d'Ivoire.

L’étude a visé toutes les personnes âgées de 18 ans et plus, vivant de façon permanente dans les ménages enquêtés et présentes au moment de l'enquête ainsi que les leaders communautaires et religieux, en vue d'obtenir leur opinion sur les VBG et les mécanismes locaux de prévention et de lutte. La collecte des données s'est faite à l'aide de deux guides d'entretien :

i. UN guide focus group adressé à la fois aux femmes et aux hommes des différentes communautés visitées. Cet outil a abordé les aspects relatifs aux perceptions sur les VBG dans la communauté, aux recours, aux mécanismes locaux de prévention et de lutte, aux normes systèmes de représentation sur les violences basées sur le genre dans les localités étudiées. Au total 10 focus-group ont été réalisés.

ii. Un guide d'entretien individuel visant les leaders communautaires et religieux, les agents de santé, des individus interrogés par la technique de quota, comporte les mêmes thématiques que le guide focus group. Au total 30 entretiens ont été réalisés. 


\section{Résultats}

\section{Perception du rôle de la femme et de la fille par les enquêtés}

La femme tout comme la fille dans le Tonkpi est un compagnon inséparable de l'homme. Elle est d'ailleurs citée comme l'élément central du ménage, en ce sens qu'elle a la charge de gérer au quotidien le ménage. En effet " c'est elle qui doit faire la cuisine, apporter l'eau aux hommes pour la boisson et pour la douche. Elle doit aussi soutenir le mari dans les travaux champêtres ». Elle est la $1^{\text {ère }}$ conseillère de l'homme et "son soutien financier » à travers le petit commerce de vivriers. Pour jouer effectivement ce rôle, la femme Yacouba doit subir une initiation (éducation aux rôles de la femme) au cours de laquelle celle-ci ressort excisée. L'excision revient en dernière analyse, l'étape achevée de l'initiation de la femme Yacouba ou Toura. Car sans cela, «elle n'est pas autorisée à se marier ou a prendre grossesse ou être admise en publique ».

Pour mieux remplir ce rôle, c'est-à-dire pour être « une bonne femme ", il importe selon les propos recueillis des focus adressés aux groupements de femmes que "la femme doit être obéissante, ne doit pas chercher à se mettre sur le même pied d'égalité que son homme, doit savoir faire la cuisine, savoir respecter son homme, savoir accueillir, éviter de défier son homme. Pour leur part, certaines attitudes doivent être évités, telles "les sorties nocturnes sans justifications valables». Aussi, ajoutent elles, "la une bonne femme ou fille doit être excisée et se marier sur l'avis de ses parents». En cas de manquement, «elle doit est corrigée». Cette « correction » qui diffère d'un homme à un autre, part de la sensibilisation à l’humiliation et peut aller jusqu’à la bastonnade voire la répudiation.

\section{Les VBG et les MGF, des outils nécessaires dans le processus de socialisation pour le peuple Yacouba?}

Au titre des types de violence contre les femmes et filles, il a été enregistré suite à nos entretiens que les femmes sont victimes de cas de bastonnade ( «frapper sa femme »), d'injures publique, quelques cas d'abus sexuel d'enfant de moins de dix ans, des coups et blessures, de viols maritaux («il ya des maris qui veulent aller toujours avec la femme, même si elle est fatiguée, il force et si la femme ne veut pas il la frappe », des violences sur la dot, des mariages forcés («c'est les parents qui choisissent le mari de la fille»). Hors mis les viols (cas rares), les violences ci-dessus citées "ne sont pas méchantes car elles s'inscrivent dans la voie de la correction ». Cela est d'autant vrai que les femmes (lors des focus) reconnaissent "qu'il y a des fois où la femme doit être battue pour être pardonnée».

Pour les répondants de sexe masculin, la plupart des violences commises sur les femmes sont des moyens de corriger celles-ci ; «il ya des 
fois où il faut frapper la femme, sans cela, elle ne changera pas, elle doit être corrigée... » Pour l'homme, la femme est reléguée au rang d'un enfant à qui l'homme doit donner des ordres, des corrections...Ainsi la plupart de ces violences prennent leur source dans les normes culturelles, dans l'interprétation des rôles, dans la représentation de la « femme ». Ces normes sont entre autre les considérations en faveur de la supériorité et des privilèges de l'homme. En effet pour le peuple Yacouba l'homme «est le chef de la maison » c’est pourquoi « la femme lui doit obéissance, respect». Partant de ce postulat, «la femme n'a jamais raison, car le masque n'a jamais tort» (entendons par masque l'homme); en outre "c'est une malédiction pour la femme et toute sa progéniture si elle revendique des amendes de réparation à son homme à la suite d'une violence subie ». Ainsi les violences sur la femme sont pour la plupart tolérées ou même justifiées par les valeurs culturelles. Il n'existe que de faibles sanctions communautaires contre les agresseurs (du cola blanc, la boisson ou un poulet). Face à cette attitude des hommes, que dira la femme, si c'est la coutume qui le dit? En effet les femmes interrogées ne disent pas le contraire: "il ya des fois où on mérite la chicotte, souvent on a des comportements qui méritent d'être frappée, l'essentiel, c'est d'être pardonner ». Tout semble se faire selon la coutume, personne ne veut $\mathrm{y}$ échapper, "de peur d'être sanctionné par les ancêtres », même les victimes. C'est le cas des mutilations génitales féminines ; en effet, ni l'homme, ni la femme ne considère l'excision comme une violence, c'est plutôt « un moyen d'humanisation de la femme, une purification de celle-ci et un moyen d'intégration sociale ». Selon les propos des femmes enquêtées, «les femmes non excisées ne sont pas propres "; c'est en vertu de toutes ces valeurs liées à l'excision qu'une femme cadre présidente d'une association féminine a été '’contrainte," à l'excision, car une fois découverte "impure », sa position sociale et son poste était menacés. Afin de maintenir cette position sociale, malgré son âge et son statut de femme mariée, " madame $X$ s'est faite excisée ». Par ailleurs, pour monter leur désaccord aux politiques de lutte contre l'excision, la population s'interrogeaient lors des entretiens « pourquoi les luttes ne prenaient pas en compte la circoncision qui est aussi une forme de mutilation».

\section{Les fondements socioculturels de la persistance de l'excision}

A l'image des peuples africains, le peuple Ivoirien accorde une grande importance à certaines vertus de la femme, telles « le respect et la soumission de la femme à son mari, la tenue vestimentaire ». Dans le Tonkpi, à l'ouest de la Côte d'Ivoire, un accent particulier est mis sur l'excision de la jeune fille pour l'intégration sociale de celle-ci. En effet l'excision constitue « la porte d'entrée et l'unique d'ailleurs » de la fille aux 
fonctions de femme dans la société Yacouba. Il est question entre autre de « contracter un mariage, porter une grossesse, accoucher au village, avoir accès à la parole sur la place publique, être leader de femme, être considérée comme une femme...». Ainsi le respect pour la femme commence par l'excision. C'est au cours des cérémonies de l'excision que l'on apprend à la femme comment tenir un foyer, comment respecter les autres membres de la société et les grandes valeurs du peuple Yacouba. Toute fille non excisée « n’a pas de respect et n'est pas une fille aux yeux du peuple Yacouba ». En clair, une fille dans un tel état est "vomie" par tous les parents des prétendants au mariage. En effet, " les parents ''Yacouba"' ne veulent pas de ces types de femmes dans leur famille", car les non excisées sont "mihomme mi-femme », elles doivent affranchir le stade de l'excision pour être " pleinement des femmes mûres, aptes au test du mariage ». D'ailleurs, toute fille non initiée (non excisée) est « à coup sûr excisée par les matrones lors des accouchements. " Au cas où l'accouchement est fait par les sages femmes, les exciseuses font leur travail à la maison lors des soins qu'elles prétendent apporter à la nouvelle maman ", en d'autres termes on " échappe jamais à l'excision au pays des Yacouba ». Les peuples du Tonkpi veillent au respect de la coutume, de peur de se voir «punir » par les ancêtres. Disentils, « on est né dans ça, on ne peut pas banaliser les pratiques de nos parents ; on a peur que les ancêtres s'en prennent à nous, on est tenu de respecter la pratique ». Pour les excisées, « il importe d'éviter d'être la risée des autres femmes ou d'être la victime d'une sorcière », car les sorcières cherches leurs proies parmi les «filles non soumises, rejetées par les parents pour une raison quelconque ». Pour leur part, " mieux vaux accepter d'être mutilée au niveau du sexe, et avoir la bénédiction des parents que de le refuser et subir la malédiction»

Outre ce raisonnement qui soutient la pratique des mutilations génitales féminines dans le Tonkpi, il est aussi admis que tout comme l'homme, la femme doit "tailler tous les débordements du sexe » pour rendre cette partie intime propre. Chez les femmes, l'excision diminue l'envie sexuel et favorise la fidélité. Elle évite à la fillette les grossesses précoces. L'objectif en dernier essor « est de garder les filles chastes jusqu'à l'âge de mariage ».

Pour parvenir à cet objectif, les populations et les exciseuses contournent les politiques des structures étatiques et ONG en charge de la lutte contre les MGF.

\section{De nouveaux comportements face à la loi d'interdiction des MGF}

Conscient des décisions de l'Etat interdisant la pratique de l'excision, surtout soucieux des risques pénaux, les populations du Tonkpi qui n'entendent pas lâcher cette pratique si «chère » à leur culture, usent de 
diverses stratégies pour atteindre leur but, celui de voir leurs progénitures filles excisées. Dès l'abord, notons que la population du Tonkpi ou tout au moins les villages (lieux des excisions) abritent moins de $1 \%$ de personnes étrangères (allochtone et allogène), (CRD, février-mars 2016). Cette caractéristique sociodémographique a permis aux détenteurs des us et coutumes de valoriser les pratiques culturelles, telle l'excision. Ainsi, toute la population hors mis les enseignants et le boutiquier (peuhl), tout genre, âge et niveau d'instruction confondu, développent des arguments valorisant l'excision, l'approuvent et souhaite sa pérennisation. Etant donné que toutes les composantes de la population sont pour la pratique (y compris les excisées et celles qui attendent à être excisée), la stratégie consiste à détourner l'attention des agents de l'Etat et ONG sur la persistance de la pratique. Pratiquement, il n'existe pas de technique extraordinaire dont usent les peuples du Tonkpi face à la politique d’éradication de l'excision. En effet, la stratégie consiste à « un renfermement sur soi », à un refus de se prononcer sur la question «tabou », c'est-à-dire l'excision. De même, chez les excisées et leurs parents, des attitudes sont tenues: interdiction de partager toute information sur «leur état d'excisée", quelque soit l'affinité, interdiction de se comporter en excisée (faire pitié, trop silencieuse), « être forte, supporter la douleur ». Aussi, faut-il noter, dans la plupart des villages, on assiste à un transfert des filles à exciser dans des villages comme «des vacancières ». Il est donc question de changement (pour la plupart) des sites de l'excision, des périodes et même des heures. Quant aux parents, ils sont tenus d'éviter toute cérémonie festives ; en clair, pas de fête au nom des excisées. Ainsi les fêtes prennent d'autres dénominations, celles de «fête d'ignames". En clair, les populations refusent de se prononcer sur toute question en rapport avec l'excision, ou quand elles en parlent, elles diront que «la pratique a existé au temps des grands pères, aujourd'hui cela ne se pratique plus ». Elles ont d'ailleurs des arguments convaincants car, te parleront des conséquences négatives de l'excision, telles les maladies en rapport avec l'excision, les avortements provoqués, les accouchements difficiles...; Par la suite elles se proposeront d'appartenir aux structures de lutte contre les MGF, les VBG. Après l'excision, les filles vaguent à leurs préoccupations ordinaires comme si rien n'était. Seules des investigations profondes permettront au socioanthropologue de constater la persistance de l'excision dans les villages. Face donc à l'excision, les populations les pratiquant développent des attitudes de complicité, de tolérance, et de dissimulation.

Toutes ces réactions importent de s’interroger sur leur niveau de connaissance des conséquences des VBG.

Les conséquences des actes de violence ne sont pas ignorées par les enquêtés, en effet la quasi-totalité reconnait par exemple que les injures 
frustrent et a des effets psychologiques. En effet, «la personne qui est tout temps insultée a honte de paraître en publique, de s'exprimer publiquement; la femme qui est mal excisée, battue court des risques de l'avortement; quand tu portes une grossesse tu peux perdre l'enfant, si les parties excisées sont mal cicatrisées la femme court le risque de la stérilité...».

\section{Conclusion}

Il ressort de cette étude que l'excision, référent de la culture du peuple du Tonkpi, conditionne encore la vie de la «femme Yacouba». Ainsi, malgré les décrets interdisant cette pratique et les risques liés à la pratique, les populations, toutes composantes confondues, complices, contournent à travers des stratégies mises en place, les agents de l'Etat et des ONG engagés dans la lutte contre les VBG. Face à cette réalité, l'approche répressive affiche des insuffisances.

\section{References:}

1. CRD, enquête sur les VBG dans le Tonkpi (février-mars 2016)

2. Enquête Démographique et de Santé et à Indicateurs Multiples 20112012

3. Guedes, A. 2004. Addressing Gender-Based Violence from the Reproductive Health/HIV Sector : A Literature Review and Analysis. Washington, DC : USAID, Bureau for Global Health. Disponible sur : http://www.prb.org/pdf04/ AddressGendrBasedViolence.pdf. Hakimi, M., E.

4. Heise, L., M. Ellsberg et M. Gottemoeller. 1999. « Ending Violence Against Women ». Population Reports XXVII (Number 4, Series L, Number 11). Disponible sur : http:// www.infoforhealth.org/pr/111/violence.pdf.

5. http://www.achpr.org/fr/instruments/declaration-on-gender-equalityin-africa/

6. http://www.unicef.org/cotedivoire/french/RESUME_CHILDPROT_ CI_NOV2011.pdf

7. La Convention sur l'élimination de toutes les formes de discrimination à l'égard des femmes(CEDEF)www.un.org/womenwatch/daw/cedaw/www.unhchr .ch/tbs/doc.nsf/Documentsfrsetfr?OpenFrameSet

8. Ministère de la Famille, de la Femme, de l'Enfant et des Affaires sociales, La Stratégie nationale de lutte contre les violences basées sur le genre (2012).

9. Opadou Koudou, Traore Fatoumata, la résistance au changement chez des femmes violentes en Côte d'Ivoire: cas des exciseuses, in 
European Scientific Journal December 2015 edition vol.11, No.35 ISSN: 1857 - 7881 (Print) e - ISSN 1857- 7431222

10. Plate Forme des VBG dans le Tonkpi, Rapport annuel de la Plate Forme des VBG dans le Tonkpi, 2015.

11. Rapport de tournée du sous-préfet de Sandougou-Soba, 05 mai 2015

12. Rapports du Comité la CEDEF:www.un.org/womenwatch/daw/cedaw

13. UNFPA (2008). Crises et violences basées sur le Genre en Côte d’Ivoire : Résultats des études et principaux défis. 